\title{
In-vitro gastrointestinal digestion of kenaf seed oil-in-water nanoemulsions
}

\begin{abstract}
The high nutrition value of kenaf seed oil has good potential to be used as functional foods or nutraceutical products. Kenaf seed oil-in-water nanoemulsions stabilised by ternary emulsifier mixtures, namely sodium caseinate, gum Arabic and Tween 20 were produced by using high pressure homogeniser. A two-stage in-vitro model was employed to investigate the bioaccessibility of bioactive compounds that is naturally present in the kenaf seed oil-inwater nanoemulsions. The changes in the antioxidants properties before and after in-vitro digestion and the structural changes during digestion were also evaluated. By comparing the digested and undigested nanoemulsions, the digested nanoemulsions had increased the total phenolic content by $71 \%$ and tocopherol content by 230\%. However, 2,2-diphenyl-1picrylhydrazyl (DPPH.) radical scavenging activity was decreased by $34 \%$ and phytosterols content was decreased by $39 \%$. The amount of free fatty acids (FFA) released from gastric digested nanoemulsions during $120 \mathrm{~min}$ of intestinal digestion was $247.7 \mu \mathrm{mol} / \mathrm{mL}$. This high release of FFA indicates good lipid digestion, which is the preliminary step for releasing and absorption of lipophilic bioactive in the small intestine. This study provides useful insights into the changes of kenaf seed oil-in-water nanoemulsions during gastrointestinal digestion.
\end{abstract}

Keyword: Kenaf (Hibiscus cannabinus L.) seed oil; High pressure homogenizer; In-vitro digestion; Bioaccessibility; Antioxidant activity; Confocal microscopy 